\title{
List of contributing authors
}

\section{Professor Lars Berglund,}

Department of Fiber and Polymer Technology, Wallenberg Wood Science Centre,

KTH Royal Institute of Technology, SE-100 44

Stockholm, Sweden,

email: blund@kth.se

\section{Professor Leif A. Carlsson,}

Department of Ocean and Mechanical

Engineering, Engineering Building 36,

Florida Atlantic University, Boca Raton,

FL 33431-0991, USA,

email: carlsson@fau.edu

Professor Douglas W. Coffin,

Department of Chemical, Paper, and

Biomedical Engineering, 64 Engineering

Building, Miami University, Oxford, $\mathrm{OH}$

45056, USA,

email: coffindw@miamiOH.edu

Professor Per-Johan Gustafsson, ${ }^{1}$

Department of Construction Sciences, Lund University, Box 118, SE-221 00 Lund, Sweden

\section{Dr. Rickard Hägglund,}

SCA, Box 716, SE-851 21 Sundsvall, Sweden, email: rickard.hagglund@sca.com

Professor Artem Kulachenko,

Department of Engineering Mechanics,

Solid Mechanics, KTH Royal Institute of

Technology, SE-100 44 Stockholm, Sweden, email: artem@kth.se

\section{Dr. Petri Mäkelä,}

Department of Engineering Mechanics, Solid Mechanics, KTH Royal Institute of

Technology, SE-100 44 Stockholm, Sweden, email: petri.makela@telia.com

\section{Professor Kaarlo Niskanen,}

FSCN, Mid Sweden University, SE-851 70

Sundsvall, Sweden,

email: kaarlo.niskanen@miun.se

\section{Dr. Mikael Nygårds,}

Department of Engineering Mechanics, Solid Mechanics, KTH Royal Institute of

Technology, SE-100 44 Stockholm, Sweden, email: mikael.nygards@billerudkorsnas.com

Professor Sören Östlund,

Department of Engineering Mechanics, Solid Mechanics, KTH Royal Institute of Technology, SE-100 44 Stockholm, Sweden, email: soren@kth.se

\section{Professor Emeritus Tetsu Uesaka,}

Department of Chemical Engineering and

FSCN, Mid Sweden University, SE-851 70

Sundsvall, Sweden,

email: tetsu.uesaka@miun.se

\footnotetext{
1 Professor Gustafsson sadly passed away during the preparation of the second edition of this book, but, prior to that, he was able to finalize Chapter 2.
} 
